\title{
Normative Values of Intracranial Translucency (IT) Thickness as an Ultrasonographic Marker for Neural Tube Defect (NTD) Screening in the Iranian Singleton Pregnancies
}

\author{
Mohammad Zare Mehrjardi, ${ }^{1,}{ }^{*}$ Elham Keshavarz, ${ }^{1}$ and Morteza Sanei Taheri ${ }^{1}$ \\ ${ }^{1}$ Department of Radiology, Shahid Beheshti University of Medical Sciences (SBMU) \\ "Corresponding author: Mohammad Zare Mehrjardi, Department of Radiology, Shahid Beheshti University of Medical Sciences (SBMU). E-mail: zare@sbmu.ac.ir
}

Received 2016 December 21; Accepted 2017 February 08.

\begin{abstract}
Background: Neural tube defects are the second most common congenital anomalies following congenital heart defects. Once NTD is detected prenatally, different options are available including pregnancy termination, and intrauterine fetal surgery. Considering these facts, prenatal screening for NTDs is becoming a part of routine fetal care. Several ultrasonographic markers have been suggested for detection of NTD during pregnancy. Intracranial translucency (IT) is a relatively new ultrasonographic marker for NTD screening during first-trimester. It actually represents the fourth cerebral ventricle, seen as an anechoic structure in the mid-sagittal plane of the fetal face posterior to the brainstem. Its absence or obliteration can be an indirect sign of open spina bifida, which is caused secondary to the caudal displacement of brain in these fetuses. In this study, we aimed to determine the normative values of this marker in the Iranian population.

Objectives: To determine the normative values of intracranial translucency (IT) thickness in the Iranian singleton pregnancies.

Methods: 230 consecutive singleton pregnancies between 11 weeks and 13 weeks+6 days of gestation were included in the study during 9 months (June 2015 to February 2016) prospectively in Mahdieh and Sarem women's hospital, Tehran, Iran. Crown-rump length (CRL), nuchal translucency (NT) thickness, fetal heart rate, and nasal bone status were evaluated by ultrasound as parts of the routine first-trimester screening for aneuploidies. Precise gestational age was determined by the fetal CRL as well. In addition, IT thickness was measured in the mid-sagittal view of the fetal head between two echogenic horizontal lines: posterior border of the brainstem anteriorly and anterior aspect of the fourth ventricle choroid plexus posteriorly (figure 1). All the measurements were performed by a radiologist with four years of experience in fetal imaging. Ethnicities other than Iranian, multiple gestation pregnancies, and pregnancies conceived by assisted reproductive technology (ART) were not included in the study initially. Other exclusion criteria were any obvious anatomic fetal anomaly in the ultrasound examination, abnormal genetic or maternal serum biomarker test result, increased NT, and presence of open NTD in the post-partum follow-up.

Results: : Of the 230 included singleton pregnancies, all of them resulted in healthy live births, except for two pregnancies terminated at 19 and 17 weeks of gestation due to severe skeletal dysplasia and an abnormal genetic test result compatible with Down syndrome, respectively. None of born babies had open NTD on post-partum follow-up. Five pregnancies were excluded from the final analysis, including a fetus with increased NT, two pregnancies with abnormal maternal serum biomarker test result (one of which confirmed to be trisomy 21), poor visualization due to maternal severe obesity in one case, and skeletal dysplasia diagnosed at 19 weeks of gestation in one of pregnancies. The median maternal age was 29 (range, 16 - 49) years. Median gestational age was 12 weeks +5 days (range, 11 weeks +2 days-13 weeks +6 days). There were 68 cases per gestational week between 11 and $11+6$ weeks, 84 cases between 12 and $12+6$ weeks, and 73 cases between 13 and $13+6$ weeks. Mean IT thickness was $1.94-0.73$ mm (95\% CI, 1.84 - 2.04) in all included pregnancies. This measurement was $1.57-0.34 \mathrm{~mm}(95 \% \mathrm{CI}, 1.49-1.65)$ for fetuses between 11 and $11+6$ weeks, 1.86 $0.29 \mathrm{~mm}$ (95\% CI, $1.80-1.92)$ for fetuses between 12 and $12+6$ weeks, and $2.12-0.41 \mathrm{~mm}$ (95\% CI, $2.02-2.22)$ for fetuses between 13 and $13+6$ weeks. Kolmogorov-Smirnov analysis demonstrated normality of IT thickness distribution in the included cases $(\mathrm{P}>0.10)$. Linear regression analysis demonstrated that there was a statistically significant positive linear association between IT thickness and gestational age $(\mathrm{rs}=0.92, \mathrm{P}=0.021)$.

Conclusions: We determined the normative values of IT thickness for the Iranian fetuses in a pilot study, which may be considered for NTD screening during the first-trimester in the national and international guidelines. It is important to determine normal measurements of different ultrasonographic and biochemistry markers nationally, since there are significant differences among various ethnicities. One should expect that IT thickness increases in a linear manner during first-trimester, and in case of its obliteration or absence open NTDs should be strongly suspected.
\end{abstract}

This is an abstract presented in the 33rd Iranian congress of radiology (ICR) and the 15th congress of Iranian radiographic science association (IRSA).

Copyright (c) 2017, Tehran University of Medical Sciences and Iranian Society of Radiology. This is an open-access article distributed under the terms of the Creative Commons Attribution-NonCommercial 4.0 International License (http://creativecommons.org/licenses/by-nc/4.0/) which permits copy and redistribute the material just in noncommercial usages, provided the original work is properly cited. 